\title{
Extramedullary Plasmacytoma of the Trachea
}

\author{
Renu Sukumaran · Rekha A. Nair • \\ Priya Mary Jacob $\cdot$ Sumod Mathew Koshy \\ Arun Peter Mathew
}

Received: 28 July 2013/Accepted: 11 September 2013/Published online: 18 September 2013

(C) Springer Science+Business Media New York 2013

\begin{abstract}
Extramedullary plasmacytomas are plasma cell tumors that occur outside the bone marrow. They constitute around $4 \%$ of all plasma cell neoplasms. The most common site of extramedullary plasmacytoma is the upper aerodigestive tract-nasal cavity, paranasal sinuses and oronasopharynx. We are presenting a case of extramedullary plasmacytoma of the trachea. Trachea is an extremely rare site of plasmacytoma. When extraosseous plasmacytoma occur in uncommon sites, the distinction from B cell lymphomas showing extensive plasmacytic differentiation can be difficult and diagnostically challenging.
\end{abstract}

Keywords Extramedullary plasmacytoma $\cdot$ MALT lymphoma $\cdot$ Tracheal tumors

\section{Introduction}

Extramedullary plasmacytoma of the trachea is an extremely rare entity. In small biopsies it is very difficult to differentiate between extramedullary plasmacytoma and mature B cell lymphomas with extensive plasmacytic differentiation. This case highlights the fact that laboratory

R. Sukumaran $(\bowtie) \cdot$ R. A. Nair · P. M. Jacob

Division of Pathology, Regional Cancer Centre, Trivandrum 695011, Kerala State, India

e-mail: renu.sukumaran@gmail.com

\section{S. M. Koshy}

Division of Imageology, Regional Cancer Centre, Trivandrum 695011, Kerala State, India

\section{A. P. Mathew}

Division of Surgical Oncology, Regional Cancer Centre,

Trivandrum 695011, Kerala State, India parameters along with immunophenotypic features at times may not help to differentiate extramedullary plasmacytoma from mature B cell lymphoma with extensive plasmacytic differentiation.

\section{Case Report}

A 39 year old male patient, who was a chronic smoker presented with dyspnoea and strider. He had history of weight loss and loss of appetite for past the 3 months. Imaging showed a soft tissue mass measuring $2.6 \times 2.5 \mathrm{~cm}$ in the posterior wall of trachea causing obstruction (Figs. 1, 2). Flexible bronchoscopic examination was done, which revealed a polypoidal mass arising from posterior wall of trachea $4 \mathrm{~cm}$ below glottis with intraluminal obstruction (Fig. 3). Biopsy from the lesion showed proliferation of sheets of plasmacytoid cells in the sub epithelial region (Figs. 4,5). With the differential diagnosis of Plasmacytoma and Extranodal marginal zone lymphoma of mucosa- associated lymphoid tissue (MALT lymphoma), we proceeded with the immunohistochemical examination. The cells were intensely positive for CD138 (Fig. 6) and showed kappa light chain restriction. CD56, CD19, CD45 and CD20 were negative. Myeloma workup was done. Blood counts were normal. Bone marrow aspirate and bone marrow biopsy showed normal morphology. Urine was negative for Bence Jones Protein and serum protein electrophoresis was normal. No lytic bone lesions noted in skeletal survey. Correlating the morphology, immunostaining and clinical parameters a differential diagnosis of extraosseous plasmacytoma vs MALT lymphoma was offered. The patient received radiotherapy. After the treatment, the patient became symptomatically better. Repeat CT scan after 1 year showed an irregular soft tissue mass in the right nasal cavity with 


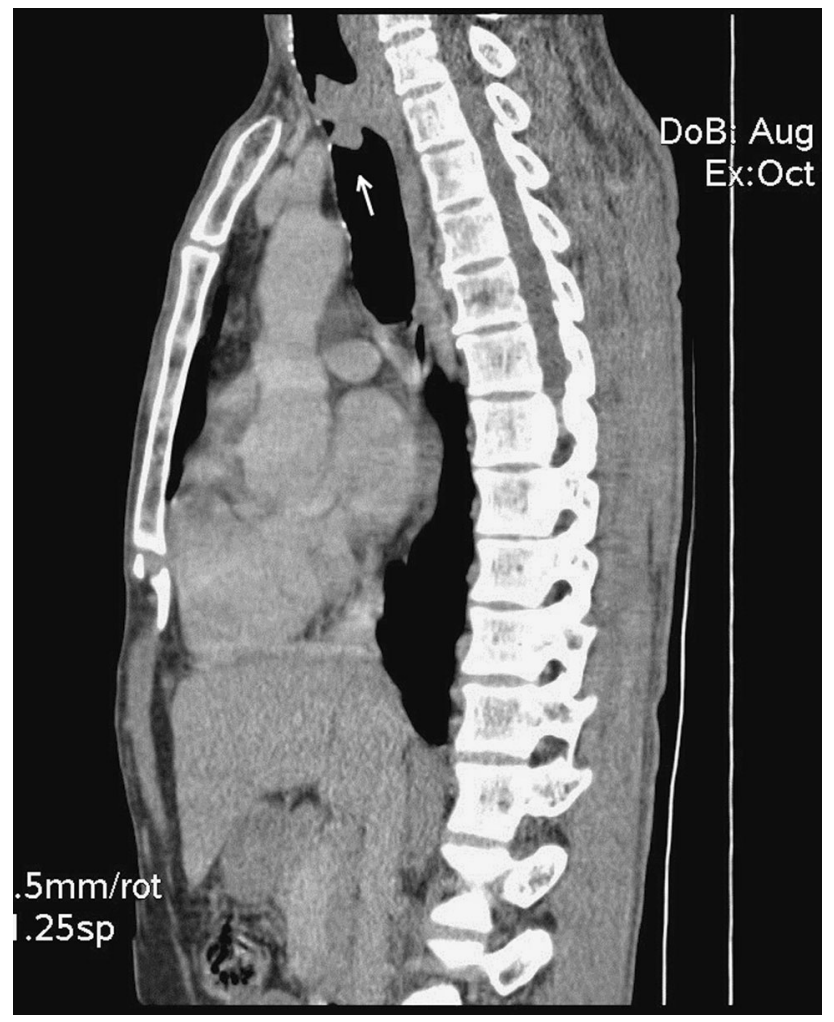

Fig. 1 Computerized tomography (sagittal section) of the thorax showing a lobulated soft tissue attenuation mass lesion in the trachea, arising from the posterior wall and protruding intra luminally

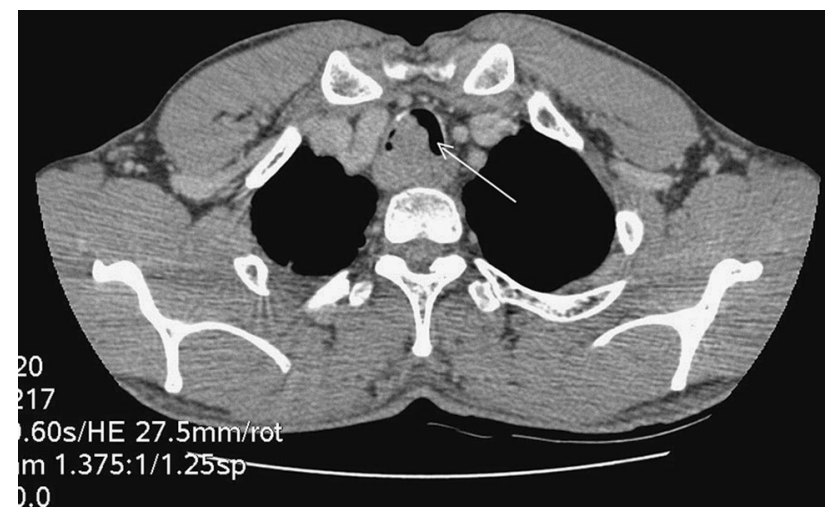

Fig. 2 Post contrast axial section showing mass lesion in the trachea, causing partial luminal compromise

destruction of hard palate measuring $1.7 \times 1.7 \mathrm{~cm}$ (Fig. 7) and an irregular soft tissue mass in the posterior wall of trachea bulging into the lumen measuring $3 \times 2 \mathrm{~cm}$. Biopsy of the mass in the nasal cavity showed sheets of plasmacytoid cells including immature and binucleate forms (Fig. 8). The immunophenotype was similar to that of the tracheal lesion. MIB1 labeling index was low (around $30 \%$ ) and EBER (EBV-encoded RNA) was negative. As there was destruction of bone, with the benefit of hindsight, the original biopsy was more likely to have been an extraosseous plasmacytoma of

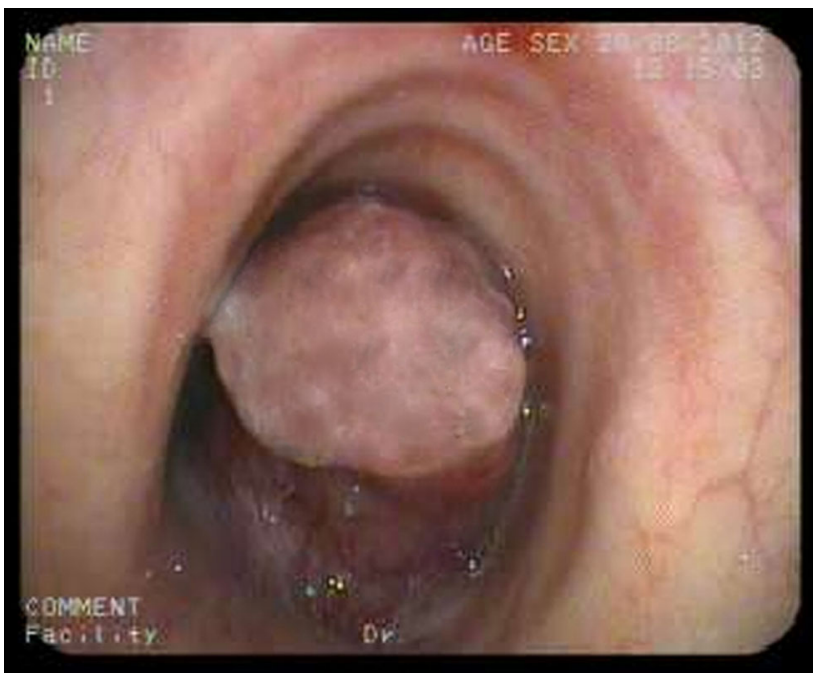

Fig. 3 Bronchoscopy showing broad based pedunculated intraluminal tracheal mass which markedly narrow the lumen

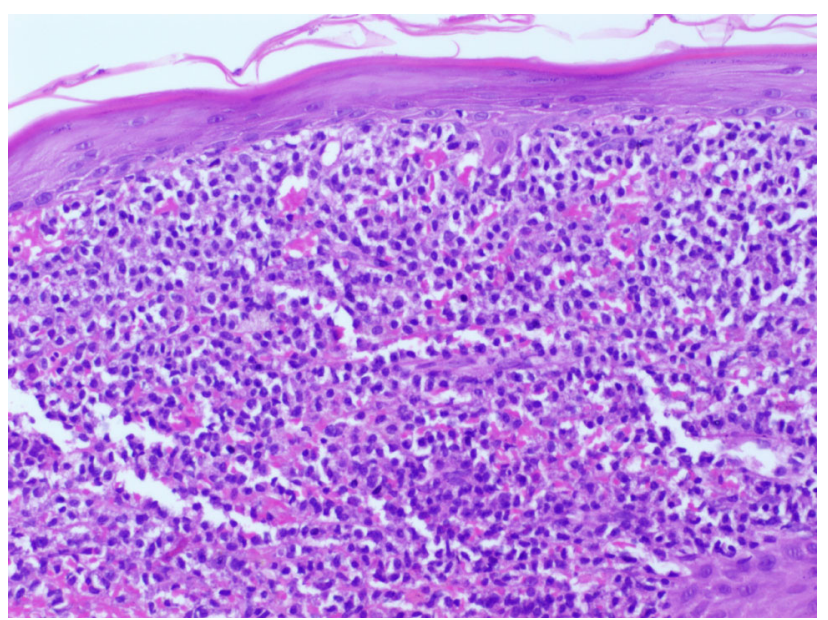

Fig. 4 Microscopy showing neoplasm beneath the intact squamous epithelium $(\mathrm{H}$ and $\mathrm{E}, \times 100)$

the trachea, rather than a MALT lymphoma. The patient was again investigated for myeloma and the workup was negative.

\section{Discussion}

Plasma cell neoplasms are characterized by neoplastic proliferation of single clone of plasma cells producing monoclonal immunoglobulins. They can present as solitary (plasmacytoma) or multiple (multiple myeloma) lesions. Solitary lesions commonly occur in the bone and are called plasmacytoma of bone. When solitary lesions occur outside the bone it is called extraosseous (extramedullary) plasmacytoma. More than $80 \%$ of cases of extramedullary plasmacytoma occur in the head and neck region-mainly 


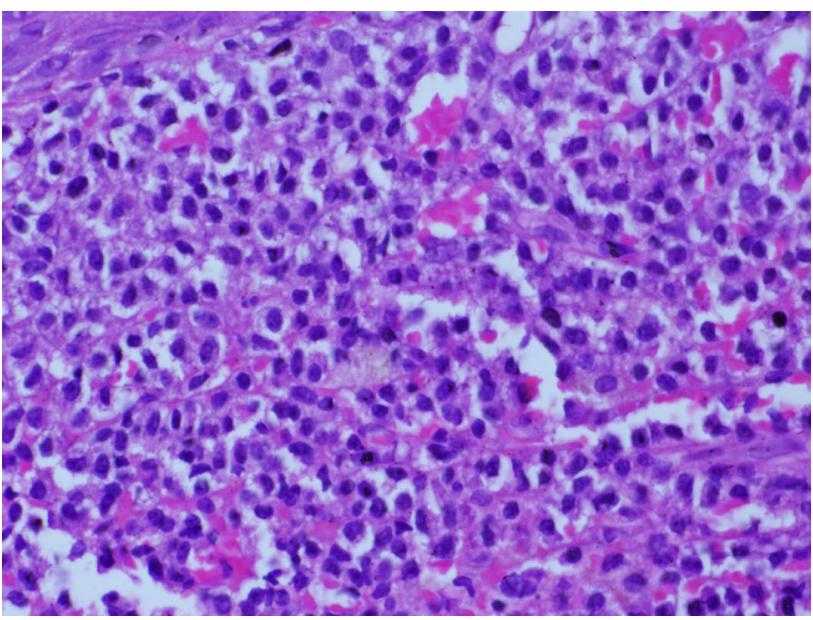

Fig. 5 Higher power showing sheets of plasmacytoid cells. (H and E, $\times 400)$

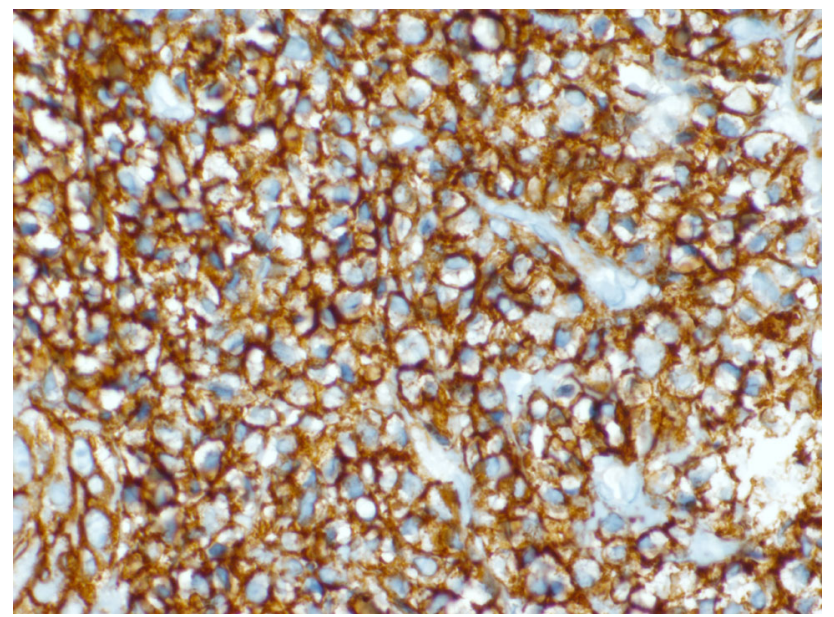

Fig. 6 Tumour cells showing intense positivity for CD138. (IHC, $\times 400$ )

in nasal cavity, paranasal sinuses and oronasopharynx [1]. Rarely extramedullary plasmacytoma can occur in the thyroid, hypopharynx, larynx, parotid gland, cervical lymph nodes and middle ear. Other areas affected include gastrointestinal tract, bladder, breast, central nervous system, testis, skin, lung parenchyma and bronchus [1-3].

Tracheal tumors as such are uncommon and constitute $0.1 \%$ of all malignancies [4]. Most common malignancies of trachea are squamous cell carcinoma and adenoid cystic carcinoma. Extramedullary plasmacytoma occurring in trachea is very rare and less than 20 cases have been reported in literature $[2,5]$. The etiology of these lesions is not well understood but viral pathogenesis and chronic irritation are suggested to be the contributing factors [6]. Clinically these patients present with nonspecific symptoms like stridor, chronic cough, dyspnoea, wheezing, hoarseness of voice or hemoptysis.

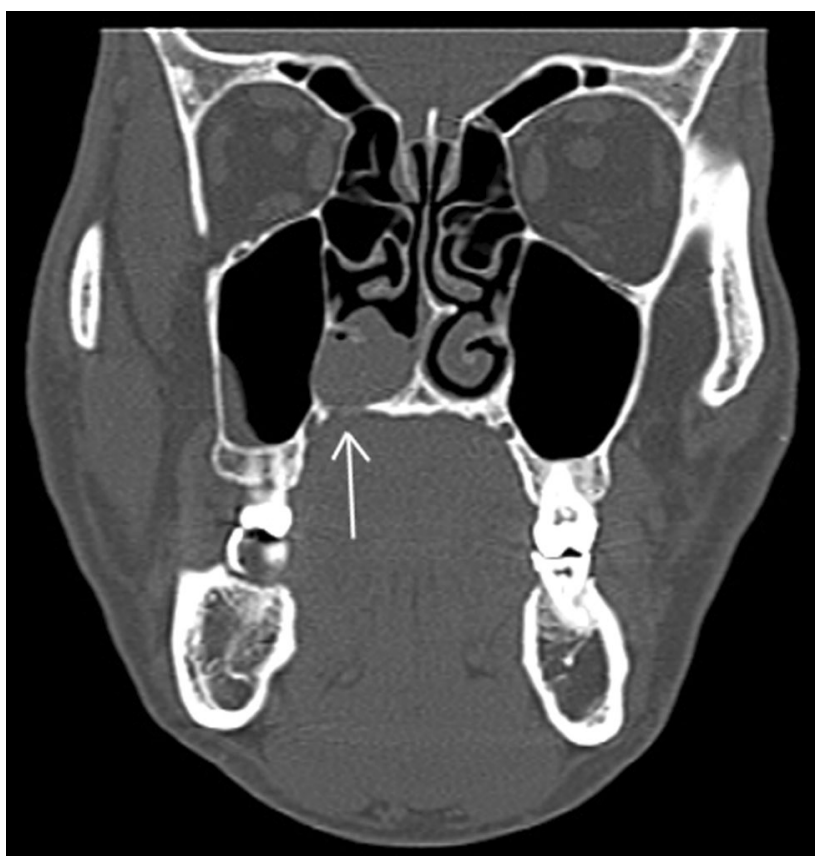

Fig. 7 Coronal $\mathrm{CT}$ sections through the paranasal sinuses (bone window) reveal a soft tissue mass in the right nasal cavity, region of inferior turbinate, with irregular destruction of adjacent hard palate

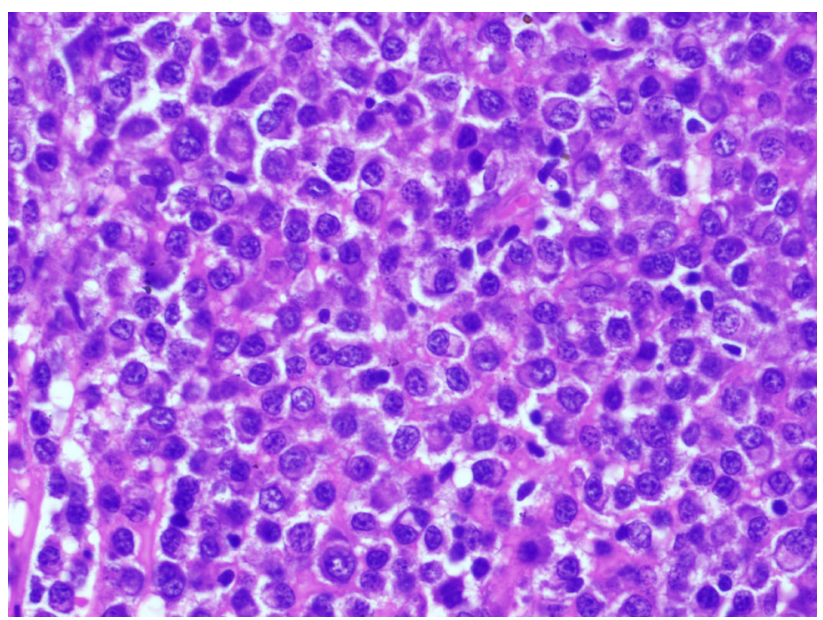

Fig. 8 Microscopy of paranasal sinus mass showing sheets of plasmacytoid cells including immature and binucleate forms. $(\mathrm{H}$ and $\mathrm{E}, \times 400$ )

Plasmacytoma is a discrete solid mass of clonal plasma cell proliferation that is immunophenotypically and cytologically identical to plasma cell myeloma, but manifests as a localized disease. Before making a diagnosis of plasmacytoma, features of multiple myeloma should be excluded by routine blood counts, skeletal survey, immunoglobulin estimation, serum protein electrophoresis and estimation of urine Bence Jones Protein.

When extensive plasmacytic differentiation is seen in small biopsies, the distinction between lymphomas with 
extensive plasmacytic differentiation and primary plasma cell dyscrasia is difficult. Plasmacytic differentiation can occur in lymphoplasmacytic lymphoma, extranodal marginal zone lymphoma of mucosa associated lymphoid tissue (MALT lymphoma), follicular lymphoma, chronic lymphocytic lymphoma and diffuse large B cell lymphoma [1]. Studies have shown that the immunophenotype of normal plasma cells and myeloma cells are different. Neoplastic plasma cells in mature B cell lymphomas will have an immunophenotype more similar to normal plasma cells than myeloma cells [7].

In the early stages of development, plasma cells express leukocyte common antigen CD45. In mature plasma cells, CD45 expression is dim or negative. Myeloma cells rarely express CD45 antigen, whereas plasma cells in B cell lymphomas are usually CD45 positive. Study by Seegmiller et al. [7] showed expression of pan B cell marker CD19 in nearly $95 \%$ cases of B cell lymphomas with plasmacytic differentiation, whereas CD19 was negative in $90 \%$ cases of myeloma. CD138 and CD38 are expressed in normal plasma cells and myeloma cells. Aberrant expression of natural killer cell marker CD56 occur in myeloma cells whereas normal plasma cells are CD56 negative $[8,9]$. Neoplastic plasma cells in mature B cell lymphomas more likely express CD19, CD45 and surface immunoglobulins like normal plasma cells while expression of CD56 will be absent [7]. Studies have shown that CD19 and CD56 are the most important immunomarkers which help to distinguish plasma cell dyscrasias from B cell lymphomas with extensive plasmacytic differentiation [7]. In the present case, both these markers were negative.

Plasmacytoma of the bone will have similar immunophenotype as that of myeloma while the immunoprofile of extraosseous plasmacytomas showed some variations. Study by Kremer et al. [10] showed aberrant expression of CD56 in more than $70 \%$ cases of multiple myeloma, but in only $10 \%$ cases of extramedullary plasmacytoma. Some extramedullary plasmacytomas showed lymphoma like phenotypeCD45 positive, CD19 positive and CD56 negative, suggesting that they may represent non-Hodgkins lymphomas with extensive plasmacytic differentiation [11].

B cell lymphomas exhibit certain immunophenotypic and molecular profiles which help in proper categorization as well as differentiation from plasmacytoma. Follicular lymphoma express B cell associated antigens (CD19, CD20, CD22, CD79a) and are usually BCL2 +, BCL6 +, $\mathrm{CD} 10+$, CD5- and CD43-. Chronic lymphocytic leukae$\mathrm{mia} /$ small lymphocytic lymphoma express CD20, CD22, CD5, CD19, CD79a, CD23, CD43 and CD 11c. In mantle cell lymphoma, the tumor cells are positive for CD5, FMC7, CD43, BCL2 and cyclin D1 but negative for CD10 and BCL6 [1]. Traslocation t $(11 ; 14)$ (q13;q32) between IGH@ and cyclin D1 genes is seen in mantle cell lymphoma whereas $\mathrm{t}(14 ; 18)(\mathrm{q} 32 ; \mathrm{q} 21)$ and $\mathrm{BCL} 2$ gene rearrangement is present in follicular lymphoma. Chromosomal translocations associated with MALT lymphomas include $\mathrm{t}(11 ; 18)(\mathrm{q} 21 ; \mathrm{q} 21), \mathrm{t}(1 ; 14)(\mathrm{p} 22 ; \mathrm{q} 32), \mathrm{t}(14 ; 18)(\mathrm{q} 32 ; \mathrm{q} 21)$ and $\mathrm{t}(3 ; 14)(\mathrm{p} 14.1 ; \mathrm{q} 32)$ [1]. $50-70 \%$ of cases of multiple myeloma have translocations involving the immunoglobulin heavy chain gene locus at 14q32. Study by Bink et al. [12] showed breaks in the $14 \mathrm{q} 32$ region in $37 \%$ of cases of extramedullary plasmacytoma, a lower rate than in multiple myeloma.

Extramedullary plasmacytoma has more favorable prognosis compared to the plasmacytoma of the bone [13]. Treatment options include surgery and/or radiation therapy. Extramedullary plasmacytoma respond well to radiation therapy $[2,6,13]$. Data from literature shows that around $60 \%$ of patients with plasmacytoma of upper aerodigestive tract had no recurrence or progression to multiple myeloma whereas $22 \%$ cases had recurrence and $16 \%$ showed progression to multiple myeloma [2, 3, 14]. Therefore even though the prognosis of plasmacytoma is better than that of multiple myeloma, a long term follow up is recommended.

\section{References}

1. Swerdlow SH, Campo E, Harris NL, Jaffe ES, Pileri SA, Stein H, Thiele J, Vardiman JW, editors. WHO classification of tumors of haematopoetic and lymphoid tissues. IARC: Lyon, France; 2008.

2. Bachar G, Goldstein D, Brown D, Tsang R, Lockwood G, PerezOrdonez B, Irish J. Solitary extramedullary plasmacytoma of the head and neck-long term outcome analysis of 68 cases. Head Neck. 2008;30:1012-9.

3. Reyhan M, Rercan F, Ergin M, Sukan A, Aydin M, Yapar F. Sonographic diagnosis of a tracheal extramedullary plasmacytoma. J Ultrasound Med. 2005;24:1031-4.

4. Papadopoulou A, Froudarakis M, Abatzoglou I, Koukourakis MI. Tracheal cancer treated with a short course of external and endoluminal radio-chemotherapy combined with cetuximab-a case report. J Contemp Brachyther. 2010;2:160-2.

5. Rai SP, Kumar R, Bharadwaj R, Panda BN. Solitary tracheal plasmacytoma. Indian J Chest Dis Allied Sci. 2003;45:269-72.

6. Lomeo PE, McDonald JE, Finneman J. Shoreline. Extramedullary plasmacytoma of nasal sinus cavities. Am J Otolaryngol. 2007;28:50-1.

7. Seegmiller AC, Xu Y, McKenna RW, Karandikar NJ. Immunophenotypic differentiation between neoplastic plasma cells in mature B cell lymphoma vs plasma cell myeloma. Am J Clin Pathol. 2007;127:176-81.

8. Ely SA, Knowles DM. Expression of CD56/neural cell adhesion molecule correlates with the presence of lytic bone lesions in multiple myeloma and distinguishes myeloma from monoclonal gammopathy of undetermined significance and lymphomas with plasmacytoid differentiation. Am J Pathol. 2002;160:1293-9.

9. Van Camp B, Durie BG, Spier C, De Waele M, Van Riet I, Vela $\mathrm{E}$, et al. Plasma cells in multiple myeloma express a natural killer cell-associated antigen:cD56 (NKH_1;Leu-19). Blood. 1990;76: 377-82.

10. Kremer M, Ott G, Nathrath M, Specht K, Stecker K, Alexiou C, et al. Primary extramedullary plasmacytoma and multiple 
myeloma: phenotypic differences revealed by immunohistochemical analysis. J Pathol. 2005;205:92-101.

11. Hussong JW, Perkins SL, Schnitzer B, Hargreaves H, Frizzera G. Extramedullary plasmacytoma : a form of marginal zone lymphoma ? Am J Clin Pathol. 1999;111:111-6.

12. Bink K, Haralambieva E, Kremer M, Ott G, Beham-Schmid C, de Leval L, et al. Primary extramedullary plasmacytoma: similarities with and differences from multiple myeloma revealed by interphase cytogenetics. Heamatologica. 2008;93:623-6.

13. Dimopoulos MA, Hamilos G. Solitary bone plasmacytoma and extramedullary plasmacytoma. Curr Treat Options Oncol. 2002;3:255-9.

14. Alexiou C, Kau RJ, Dietzfelbinger H, Kremer M, Spiess JC, Schratzenstaller B, et al. Extramedullary plasmacytoma: tumor occurrence and therapeutic concepts. Cancer. 1999;85:2305-14. 